\title{
$\mathrm{AIP} \mid \begin{aligned} & \text { Physics of } \\ & \text { Fluids }\end{aligned}$
}

\section{Singlemode saturation of a linearly unstable plasma}

C. Burnap, M. Miklavi, B. L. Willis, and P. F. Zweifel

Citation: Physics of Fluids (1958-1988) 28, 110 (1985); doi: 10.1063/1.865190

View online: http://dx.doi.org/10.1063/1.865190

View Table of Contents: http://scitation.aip.org/content/aip/journal/pof1/28/1 ?ver=pdfcov

Published by the AIP Publishing

\section{Articles you may be interested in}

A nonlocal singlemode amplification process using linear optics

AIP Conf. Proc. 1110, 161 (2009); 10.1063/1.3131296

Nonlinear saturation of singlemode trapped electron instability

Phys. Fluids 28, 3287 (1985); 10.1063/1.865326

Nonlinear saturation of singlemode trapped ion instability

Phys. Fluids 28, 1077 (1985); 10.1063/1.865400

Linear polarization in birefringent singlemode fibers

Appl. Phys. Lett. 33, 699 (1978); 10.1063/1.90534

Model equations for mode coupling saturation in unstable plasmas

Phys. Fluids 16, 855 (1973); 10.1063/1.1694437

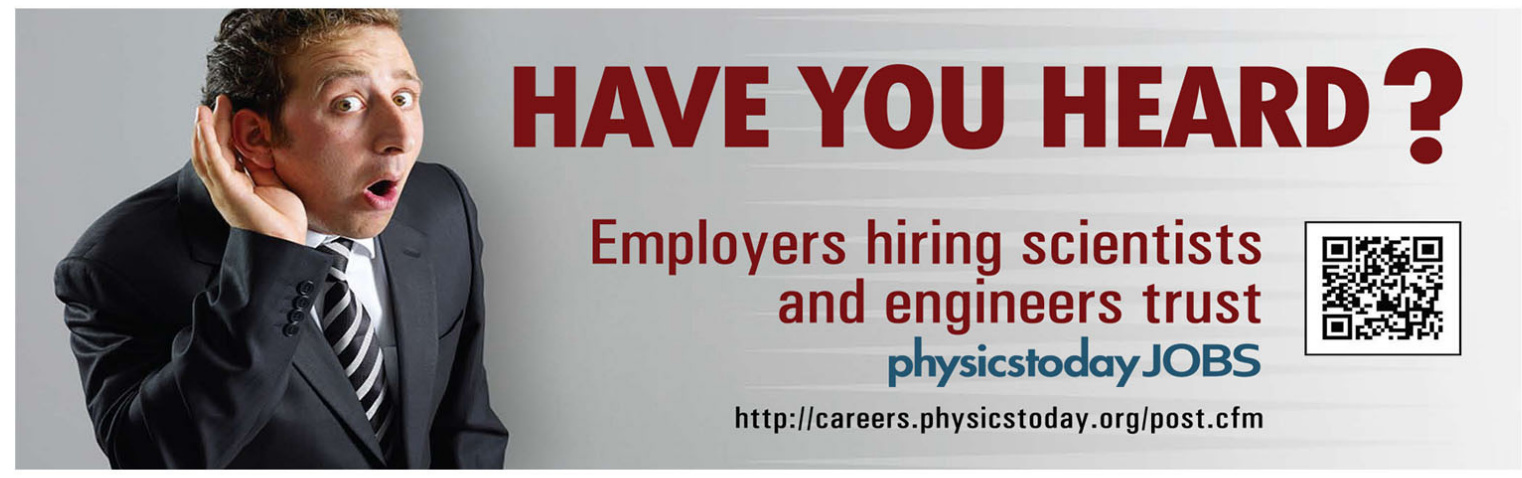




\title{
Single-mode saturation of a linearly unstable plasma
}

\author{
C. Burnap, ${ }^{\text {a) }}$ M. Miklavcic, B. L. Willis, and P. F. Zweifel \\ Laboratory for Transport Theory and Mathematical Physics, Virginia Polytechnic Institute and State \\ University, Blacksburg, Virginia 24061
}

(Received 27 December 1983; accepted 28 August 1984)

\begin{abstract}
The nonlinear oscillations of an electron plasma described by the collisionless Vlasov equation are studied using a perturbation technique previously applied by Simon and Rosenbluth [Phys. Fluids 19, 1567 (1976)]. It is proved by a characteristic argument that the plasma is globally stable, so that Bogoliuboff's method of "secular regularization" is applicable. Assuming the plasma is confined in a box, and that only the lowest mode is unstable, it is shown that the "eigenmode dominance" approximation of Simon and Rosenbluth fails to conserve energy, but that energy and momentum conservation can be regained by considering interaction between the discrete and continuum modes. A formula is derived for the amplitude and phase of the saturated nonlinear oscillations. In a subsidiary result, it is shown that nonlinear effects damp the steady-state oscillations predicted by linearized theory for some stable plasmas.
\end{abstract}

\section{INTRODUCTION}

It is well known that plasma instabilities may be induced either by irregular spatial configurations (e.g., Rayleigh-Taylor instability) or by deviation from an equilibrium Maxwellian distribution in velocity (e.g., two-stream or bump-on-tail instabilities). The latter are generally studied by analyzing the stability of solutions to the Vlasov (collisionless Boltzmann) equation.

The linearized version of the Vlasov equation has been studied by a number of authors ${ }^{1-5}$ who have been able to calculate the deviation, $f(\mathbf{r}, \mathbf{v}, t)$, of the electron density from the equilibrium distribution $F(v)$. The solution consists of an integral over the continuous spectrum $(-\infty, \infty)$ which, in general, decays time-asymptotically to zero (Landau damping) plus one or more eigenvalue contributions of the form $e^{-i k v_{i} t}$, where $k$ is the spatial Fourier transform variable and the eigenvalue $v_{i}$ is a zero of the plasma dispersion function

$$
\Lambda(z)=1-\frac{\omega_{p}^{2}}{k^{2}} \int_{-\infty}^{\infty} \frac{F^{\prime}(v) d v}{v-z} .
$$

Here,

$$
\omega_{p}=\left(4 \pi N_{0} e^{2} / m\right)^{1 / 2}
$$

is the plasma frequency, $N_{0}$ is the plasma density, and $e$ and $m$ the electron charge and mass. The function $F^{\prime}$ in Eq. (1a) represents the derivative of the equilibrium distribution $F(v)$ introduced earlier.

If $\boldsymbol{A}$ has any real zeros, we can see that the asymptotic solution to the linearized Vlasov equation undergoes steadystate oscillations (with an amplitude determined by the initial perturbation from equilibrium). On the other hand, since zeros of $\boldsymbol{A}$ occur in complex conjugate pairs, the presence of an eigenvalue with nonvanishing imaginary part guarantees the linear instability of the plasma.

It is accepted that linearized equations can give completely misleading information about stability; for example, a linearly unstable amplitude will grow to a point where the linearization approximation is no longer valid, at which point the stability question is very uncertain. The Vlasov equation is an excellent example of this phenomenon, since

\footnotetext{
a) Department of Mathematics, University of North Carolina, Charlotte, North Carolina 28223.
}

all solutions are globally stable (i.e., bounded functions of time) and, furthermore, a system that linearized theory predicts will undergo asymptotic steady-state oscillations, does not oscillate at all according to the full nonlinear equation. The proof of global stability is trivial. It follows from the fact that the (full) solution $g(r, v, t)$ of the Vlasov equation is constant along characteristics. ${ }^{6}$ Thus, the solution must be a bounded function of time, otherwise it would become infinite along every characteristic, which is impossible. The proof that nonlinear effects damp steady-state linear oscillations is presented in Sec. III.

Apparently, global stability is not sufficient for the designers of thermonuclear reactors. Rather, they would like to know that small deviations from the equilibrium distribution remain small (local stability); global stability-the sup condition-would permit growth up to the order of the ambient equilibrium distribution. Thus considerable effort has been exerted through the years to study the asymptotic amplitudes and oscillation frequency resulting from an initial perturbation of the solution to the full nonlinear equation. ${ }^{7-9}$

The method we adopt to study this problem is based on previous work of Simon and Rosenbluth. ${ }^{10}$ The model considered is that of a plasma with a weak linear instability (as, for example, a gentle "bump-on-tail"). Specifically, the plasma is confined in a box of length $2 \pi$ and subject to periodic boundary conditions. This gives rise to spatial modes of the form $e^{i k x}, k=0, \pm 1, \pm 2, \ldots$. We assume only a single spatial mode $k=n$ is present, i.e., the plasma dispersion function $\Lambda$ has a zero $v_{0}$ only for $k=n$ and not for any other value of $k$. Furthermore, for electron density $N=N_{0}$, we assume $v_{0}$ is real, i.e., the plasma is linearly stable. The density is now changed to $N_{0}(1+\Delta)$, where $\Delta<1$, and the eigenvalue moves off-axis so that the plasma becomes linearly unstable. Since we know from global stability that the solution to the nonlinear equation will saturate at some amplitude $\gamma$ and oscillate with some frequency $v$, the question is to calculate these quantities.

As did Simon and Rosenbluth ${ }^{10}$ (following the procedure of Ref. 9), we completely ignore the continuum, treating only the linear mode corresponding to $v_{0}$. This procedure, sometimes referred to as the assumption of "eigenvalue dominance," is based on the presumption that even in the 
nonlinear regime, the continuum will enjoy Landau damping ${ }^{11}$ so that asymptotically only the discrete eigenvalues will dominate.

The mathematical technique is based on a perturbation series in a small parameter $\epsilon$. It turns out that $\epsilon \sim \Delta^{1 / 2}$, where $\Delta$ is the density perturbation introduced earlier. Incidentally, in Ref. 10, and elsewhere, this proportionality is taken for granted. Since this more or less automatically predicts that the saturated amplitude will be proportional to $\Delta^{1 / 2}$, we have felt it would be worthwhile to prove the relationship between $\epsilon$ and $\Delta$. This we do in Sec. III.

As in Ref. 10, secular terms (terms that diverge with $t$ ) arise at every step of the perturbation expansion. Global stability guarantees that these terms cannot appear, and so the standard Bogoliuboff procedure of secular regularization is followed. Our solutions, as in Ref. 10, are ambiguous because of highly singular behavior on the real axis. For this reason we regularize by solving the equation "off axis," $v_{0} \rightarrow v_{0} \pm i \eta,\left(v_{0}\right.$, we recall, is real $)$ and take the limit $\eta \rightarrow 0$. This procedure leads to both the saturated amplitude and the nonlinear frequency shift. Auerbach ${ }^{12}$ has shown that this regularization procedure is equivalent to introducing a collision term into the Vlasov equation (and later taking the limit as the collision frequency tends to zero). Specific collision models leading to a similar result have been considered by other authors, e.g., a modified Fokker-Planck model of the type ${ }^{13}$ :

$$
\left(\frac{\partial f}{\partial t}\right)_{\text {coll }}=-(\alpha+\beta v) \frac{\partial f}{\partial v} .
$$

Similar results have been found for other models. ${ }^{14-16} \mathrm{Al}-$ though different collision models will lead to different regularizations, we show, in Sec. III, that the saturated amplitude and nonlinear frequency shift are independent of regularization and, hence, of presumed scattering models.

Our paper differs from Ref. 10 in two important aspects. First we believe our analysis is considerably easier to follow, as we dispense with long and complicated expressions involving Schwartz distributions (until the very end, when we need only some simple results concerning canonical regularization of locally nonintegrable functions). This is more than an aesthetic point. If we stay "on-axis" throughout the analysis, indeterminate coefficients are introduced into the solution as multipliers of delta functions. Such terms do not appear in the procedure we adopt.

More important, perhaps, is the fact that our results, i.e., the nonlinear frequency shift and saturation amplitude, differ from those of Ref. 10. Perhaps numerical comparisons should be carried out.

\section{THE BASIC METHOD}

For simplicity, we consider only a one-dimensional plasma. Let $f(x, v, t)$ represent the deviation of the density from the equilibrium value $F(v)$. Then, assuming immovable background ions, the 1-D Vlasov equation can be written as

$$
\frac{\partial f}{\partial t}+v \frac{\partial f}{\partial x}+\frac{e}{m} \frac{\partial \phi}{\partial x} F^{\prime}+\frac{e}{m} \frac{\partial \phi}{\partial x} \frac{\partial f}{\partial v}=0,
$$

while the electric potential $\phi$ obeys

$$
\frac{\partial^{2} \phi}{\partial x^{2}}=4 \pi N e \int_{-\infty}^{\infty} f(x, v, t) d v .
$$

We look for a solution of Eqs. (2) of the form

$$
f(x, v)=\sum_{n=-\infty}^{\infty} f_{n}(v) e^{i n x}
$$

For compactness, we will frequently write $\int g$ instead of $\int_{-\infty}^{\infty} g(v) d v$. Periodicity of $\phi$ and $\partial \phi / \partial x$ gives us

$$
\begin{aligned}
\frac{e}{m} \frac{\partial \phi}{\partial x} & =-i \omega_{p}^{2}(1+\Delta) \sum_{\substack{n=-\infty \\
n \neq 0}}^{\infty} \frac{1}{n}\left(\int f_{n}\right) e^{i n x} \\
& \equiv(1+\Delta) L(f) .
\end{aligned}
$$

We define a linear map $C$ by

$$
C\left(\sum f_{n} e^{i n x}\right)(x, v)=\sum_{\substack{n=-\infty \\ n \neq 0}}^{\infty}\left(i n v f_{n}(v)-i \frac{\omega_{p}^{2}}{n} F^{\prime}(v) \int f_{n}\right) e^{i n x}
$$

Thus we obtain our basic equation:

$$
\frac{\partial f}{\partial t}+C(f)+\Delta L(f) F^{\prime}+(1+\Delta) L(f) \frac{\partial f}{\partial v}=0 .
$$

We now introduce an asymptotic expansion in $\epsilon$. This expansion is defined by ${ }^{17}$

$$
\begin{aligned}
& s=\sum_{j=0}^{\infty} t \alpha_{j} \epsilon^{j}, \quad \alpha_{0}=1, \\
& \Delta=\sum_{j=0}^{\infty} \beta_{j} \epsilon^{j}, \\
& f=\sum_{j=0}^{\infty} \epsilon^{j+1} f^{(j)} .
\end{aligned}
$$

Here the $\alpha_{j}$ are the nonlinear frequency shifts. Inserting Eqs. (4) into Eq. (3) and equating coefficients of equal powers of $\epsilon$, an equation of the form

$$
\frac{\partial f}{\partial s}+C(f)=h z^{k}, \quad k \in Z, \quad k \neq 0
$$

occurs at each order, where

$$
z=\exp \left(i n x-i n v_{0} s\right) \text {. }
$$

If we let $f=\left(s \phi_{1}+\phi_{2}\right) z^{k}, \quad \phi_{i}=\phi_{i}(v), \quad$ and $g_{n}=\omega_{p}^{2} / n^{2}\left(v-v_{0}\right)$, then a straightforward calculation gives

$$
\phi_{1}=\frac{g_{n} F^{\prime}}{k^{2}} \int \phi_{1}
$$

and

$$
n\left(v-v_{0}\right) \phi_{2}-\frac{\omega_{P}^{2}}{k^{2} n} F^{\prime} \int \phi_{2}=\frac{h-\phi_{1}}{i k} .
$$

Integrating (6) shows that for some constant $a$,

$$
\phi_{1}= \begin{cases}a g_{n} F^{\prime} & k= \pm 1, \\ 0 & |k|>1,\end{cases}
$$

where $g_{n} F^{\prime}$ is a Case eigenfunction. Thus, as is well known, secular behavior can occur only if $k= \pm 1$. We impose the condition that there are no secular terms in $f$, i.e., $a=0$. Setting $\phi_{1}=0$ in (7) requires

$$
\int g_{n} h=0 \text { if } k= \pm 1
$$

If (8a) holds, solving (7) for $\phi_{2}$ we obtain 


$$
f= \begin{cases}\frac{n}{i \omega_{p}^{2} k} g_{n} h z, & k= \pm 1, \\ \frac{n}{i k \omega_{p}^{2}}\left(\frac{g_{n} F^{\prime}}{k^{2}-1} \int g_{n} h+g_{n} h\right) z^{k}, & |k|>1 .\end{cases}
$$

In the above analysis, numerous integrals occur that are not defined for $v_{0}$ real. As stated in the introduction, we therefore assume the eigenvalue is of the form $v_{0} \pm i \eta$ and eventually take the limit $\eta \rightarrow 0+$. The $f$ 's one obtains in this way are distributional solutions of Eq. (5) with $v_{0}$ real. (The existence of classical solutions would severely restrict the allowable equilibrium $F$ 's.) Moreover, Eqs. (8) would not be correct if $h$ involved delta functions or derivatives thereof.

\section{SOLUTIONS $f^{(0)}, f^{(1)}$, and $f^{(2)}$}

To lowest order, Eq. (3) is

$$
\frac{\partial f^{(0)}}{\partial s}+C\left(f^{(0)}\right)+\beta_{0} F^{\prime} L\left(f^{(0)}\right)=0 \text {. }
$$

The dispersion function for this equation is easily verified to be

$$
\Lambda=1-\frac{\left(1+\beta_{0}\right) \omega_{p}^{2}}{n^{2}} \int_{-\infty}^{+\infty} \frac{F^{\prime}(v)}{v-z} d v .
$$

Unless $\beta_{0}=0, \Lambda$ will have a nonreal zero, i.e., secular behavior results unless $\beta_{0}=0$. After setting $\beta_{0}=0$, the solution to Eq. (9) is well known ${ }^{1-5}$ :

$$
f^{(0)}=\gamma g_{n} F^{\prime} z+\text { c.c., }
$$

where $\gamma$ is a complex amplitude and c.c. indicates complex conjugate. Note that $f^{(0)}$ is a well-behaved function since $F^{\prime}\left(v_{0}\right)=0$.

We now show $\Delta \sim \epsilon^{2}$ by proving $\beta_{1}=0$ (recall that $\beta_{0}=0$; see preceding discussion). In order $\epsilon^{2}$, Eq. (3) yields

$$
\begin{aligned}
\frac{\partial f^{(1)}}{\partial s}+C\left(f^{(1)}\right)= & -\alpha_{1} \frac{\partial f^{(0)}}{\partial s}-L\left(f^{(0)}\right) \frac{\partial f^{(0)}}{\partial v} \\
& -\beta_{1} F^{\prime} L\left(f^{(0)}\right) .
\end{aligned}
$$

It is convenient to define $\psi_{1}=g_{n} F^{\prime}, \psi_{2}=g_{n} \psi_{1}^{\prime}$, and $\psi_{3}=g_{n} \psi_{2}^{\prime}$. The right-hand side of Eq. (12) is then

$$
\left[i n \alpha_{1} v_{0} \gamma \psi_{1}+\left(i \omega_{p}^{2} / n\right) \beta_{1} \gamma F^{\prime}\right] z+\left[\left(i \omega_{p}^{2} / n\right) \gamma^{2} \psi_{1}^{\prime}\right] z^{2}+\text { c.c. }
$$

Because the right-hand side includes a term proportional to $z$ we obtain a growing term in $f^{(1)}$ unless [see Eq. (8a)]

$$
\int i n \alpha_{1} v_{0} \gamma g_{n} \psi_{1}+\int \frac{i \omega_{p}^{2}}{n} \beta_{1} \gamma g_{n} F^{\prime}=0,
$$

which implies

$$
n \alpha_{1} v_{0} \int g_{n} \psi_{1}+\frac{\omega_{p}^{2}}{n} \beta_{1}=0 .
$$

The function $g_{n} \psi_{1}$ is unbounded at $v=v_{0}$, so we replace $v_{0}$ by $v_{0} \pm i \eta$ and evaluate the limit $\eta \rightarrow 0+$. The result is

$$
n \alpha_{1} v_{0}\left(\mathrm{CR} \int \psi_{2} \pm i \pi F^{\prime \prime}\left(v_{0}\right)\right)+\frac{\omega_{p}^{2}}{n} \beta_{1}=0,
$$

where $\mathrm{CR}$ indicates the usual canonical regularization. ${ }^{18}$ Equation (15) implies $\alpha_{1}=\beta_{1}=0$, and hence $\Delta$ is of order $\epsilon^{2}$.
We now turn our attention to the highest-order solutions $f^{(1)}$ and $f^{(2)}$. From Eq. (12), using Eq. (8b), we obtain

$$
f^{(1)}=\gamma^{2} \theta z^{2}+\text { c.c., }
$$

where $\theta$ is defined to be

$$
\theta=\frac{1}{6} \psi_{1} \int \psi_{2}+\frac{1}{2} \psi_{2}
$$

Thus equating the coefficients of $\epsilon^{3}$ in Eq. (3) gives

$$
\frac{\partial f^{(2)}}{\partial s}+C\left(f^{(2)}\right)=u_{3} z^{3}+u_{1} z+\text { c.c., }
$$

with

$u_{3}=\frac{i \omega_{p}^{2}}{2 n} \gamma^{3}\left(\psi_{1}^{\prime} \int \theta+2 \theta^{\prime}\right)$,

$u_{1}=\frac{i \omega_{p}^{2}}{2 n} \gamma\left(\frac{2 v_{0} n^{2} \alpha_{2}}{\omega_{p}^{2}} \psi_{1}+2 \beta_{2} F^{\prime}-2|\gamma|^{2} \theta^{\prime}+|\gamma|^{2} \psi_{1}^{\prime} \int \theta\right)$.

Absence of secularity requires

$$
\begin{aligned}
& \gamma\left[\frac{2 v_{0} n^{2} \alpha_{2}}{\omega_{p}^{2}} \int g_{n} \psi_{1}+2 \beta_{2}\right. \\
& \left.\quad+\frac{1}{3}|\gamma|^{2}\left(\int \psi_{2}\right)^{2}-|\gamma|^{2} \int \psi_{3}\right]=0,
\end{aligned}
$$

and $f^{(2)}$ is given by

$f^{(2)}=2 \operatorname{Re}\left[\frac{n z^{3}}{3 i \omega_{p}^{2}}\left(\frac{\int g_{n} u_{3}}{8} \psi_{1}+g_{n} u_{3}\right)+\frac{n z}{i \omega_{p}^{2}} g_{n} u_{1}\right]$.

Recall that we define the integrals in Eqs. (18) by replacing $v_{0}$ with $v_{0} \pm i \eta$ and letting $\eta \rightarrow 0+$. Since secular regularization requires $\operatorname{Im} \alpha_{2}=0$ (or else we will obtain exponentially growing solutions), taking the real and imaginary parts of Eq. (18) yields, for $\gamma \neq 0$ and $v_{0} \rightarrow v_{0}+i \eta$,

$$
\begin{gathered}
\frac{2 v_{0} \alpha_{2} n^{2}}{\omega_{p}^{2}}\left(\mathrm{CR} \int g_{n} \psi_{1}\right)+2 \beta_{2}+\frac{|\gamma|^{2}}{3}\left[\left(\mathrm{CR} \int \psi_{2}\right)^{2}\right. \\
\left.-\left(\frac{\pi \omega_{p}^{4}}{2 n^{4}} F^{\prime \prime \prime}\left(v_{0}\right)\right)^{2}\right]-|\gamma|^{2}\left(\mathrm{CR} \int \psi_{3}\right)=0,
\end{gathered}
$$

and

$$
\begin{gathered}
\frac{2 v_{0} \alpha_{2} \omega_{p}^{2}}{n^{2}} F^{\prime \prime}\left(v_{0}\right)+\frac{1}{3}|\gamma|^{2}\left(\mathrm{CR} \int \psi_{2}\right) \frac{\omega_{p}^{4}}{n^{4}} F^{m}\left(v_{0}\right) \\
-\frac{|\gamma|^{2} \omega_{p}^{6}}{8 n^{6}} F^{(5)}\left(v_{0}\right)=0 .
\end{gathered}
$$

Here $\mathrm{CR}$ indicates the usual canonical regularization. ${ }^{18}$ In particular,

$$
\begin{aligned}
& \mathrm{CR} \int_{-\infty}^{+\infty} g_{n} \psi_{1}=\frac{\omega_{p}^{4}}{n^{4}} \int_{0}^{\infty} \frac{d v}{v^{2}}\left[F^{\prime}\left(v_{0}+v\right)+F^{\prime}\left(v_{0}-v\right)\right], \\
& \mathrm{CR} \int_{-\infty}^{+\infty} \psi_{2}=\frac{\omega_{p}^{4}}{n^{4}} \int_{0}^{\infty} \frac{d v}{v}\left(\frac{F^{\prime}\left(v_{0}+v\right)-F^{\prime}\left(v_{0}-v\right)}{v}\right)^{\prime},
\end{aligned}
$$

$\mathrm{CR} \int_{-\infty}^{+\infty} \psi_{3}=\frac{\omega_{p}^{6}}{n^{6}} \int_{0}^{\infty} \frac{d v}{v}\left[\frac{1}{v}\left(\frac{F^{\prime}\left(v_{0}+v\right)-F^{\prime}\left(v_{0}-v\right)}{v}\right)^{\prime}\right]^{\prime}$

Equations (19) may be solved simultaneously for $\alpha_{2}$ and $|\gamma|^{2}$, giving 


$$
\alpha_{2}=\frac{3 \omega_{p}^{4} F^{(5)}\left(v_{0}\right)-8 n^{2} \omega_{p}^{2} F^{(3)}\left(v_{0}\right)}{24 n^{4} D} \beta_{2},
$$

and

$$
|\gamma|^{2}=\left[2 F^{\prime \prime}\left(\nu_{0}\right) / D\right] \beta_{2},
$$

where

$$
\begin{aligned}
D= & \left(\mathrm{CR} \int g_{n} \psi_{1}\right)\left[\frac{1}{3} F^{(3)}\left(v_{0}\right)\left(\operatorname{CR} \int \psi_{2}\right)-\frac{\omega_{p}^{2}}{8 n^{2}} F^{(5)}\left(v_{0}\right)\right] \\
& +F^{\prime \prime}\left(v_{0}\right)\left[\left(\operatorname{CR} \int \psi_{3}\right)+\frac{1}{3}\left(\frac{\pi \omega_{p}^{4}}{2 n^{4}} F^{(3)}\left(v_{0}\right)\right)^{2}\right. \\
& \left.-\frac{1}{3}\left(\operatorname{CR} \int \psi_{2}\right)^{2}\right] .
\end{aligned}
$$

Although $\alpha_{2}$ and $|\gamma|^{2}$ have been regularized by going above the axis $\left(v_{0} \rightarrow v_{0}+i \eta\right)$, the results are independent of regularization, except in the case of principal value regularization. The demonstration of this fact is trivial. The most general regularization is a convex combination of above and below the axis regularizations. Denoting the value of $\int u_{1} g_{n}$ resulting from $v_{0} \rightarrow v_{0}+i \eta$ by $K+i J$, and noting that the below axis regularization is the complex conjugate of the above axis regularization, we have

$$
0=a(K+i J)+b(K-i J), \quad a+b=1 .
$$

Taking real and imaginary parts yields

$$
\begin{aligned}
& 0=K-\operatorname{Im}(a-b) J, \\
& 0=\operatorname{Re}(a-b) J .
\end{aligned}
$$

Therefore, except in the case $\operatorname{Re}(a-b)=0$, i.e., the principal value regularization, the only solution to the above equations is $K=J=0$ as was required in Eqs. (19).

It should be noted that, to leading order in $\epsilon$, all regularizations result in the same solution $f^{(0)}$. In second order we obtain a set of solutions, $f^{(1)}$, each of which is unbounded as a function of velocity. However, these solutions can be integrated against a smooth kernel in order to construct a smooth solution. This superposition is permitted in this order since with $f^{0}$ fixed, the equation that determines $f^{(1)}$ is linear. Higher-order solutions can be dealt with in the same way and thus positivity of the complete solution (including the equilibrium distribution) can be maintained.

Also note that $\gamma=0$ is always a solution of Eq. (18a), and it is the only solution if (21a) gives $|\gamma|^{2}<0$, or $D=0$. In this case $\alpha_{2}$ is not determined to this order. Moreover, we see that $\gamma=0$ if $F^{\prime \prime}\left(v_{0}\right)=0$. However, if $F^{\prime \prime}\left(v_{0}\right)=0$ the plasma is linearly stable to order $\epsilon^{3}$ (see Ref. 19). The absence of oscillations $(\gamma=0)$ for a linearly stable plasma is not surprising in light of the following result.

To treat the case of a linearly stable plasma, i.e., $\Delta=0$, we need only set $\beta_{2}=0$. Since $|\gamma|$ is proportional to $\beta_{2}$, this implies that a linearly stable plasma will not oscillate.

\section{THE EFFECT OF THE CONTINUUM}

For the parameter values considered in this paper, the point eigenvalue lies very close to the continuous spectrum, i.e., the real line. Nonetheless, the model we have chosen, eigenvalue dominance, ignores the continuum entirely, on the grounds that Landau damping makes it unimportant after a short time. This assumption is certainly reasonable for linear terms in the equation. However, interference (cross terms) between the point and continuous spectrum and, indeed, between different portions of the continuous spectrum can be important, as we now show. In particular, such interference can reinstate energy conservation that is not satisfied in the eigenvalue dominance model. Since we have not obtained numerical results yet, we do not present the full analytical computation in this section, giving instead the outline of the argument.

According to the model of eigenvalue dominance, which we have adopted in this paper, the equilibrium distribution (i.e., the portion of the distribution function indepedent of $x$ ) is not modified by nonlinear effects. The reason for this is quite clear-such modifications can arise only from the terms [of Eq. (3)] $L(f)\left(\partial f^{*} / \partial v\right)+L\left(f^{*}\right)(\partial f / \partial v)$. These terms sum to zero in the limit $\eta \rightarrow 0$, leaving the equilibrium distribution unchanged. Our analysis differs from that of Simon and Rosenbluth, ${ }^{10}$ who arrange for such terms to have a nonzero contribution in what seems to us to be a somewhat contrived manner.

The nonlinear oscillations, by virtue of the periodic boundary conditions, have zero kinetic energy. However, they do carry electric field energy given by

$$
W_{E}=\frac{1}{8 \pi} \int_{0}^{2 \pi} E^{2} d x=\frac{2 \pi}{n^{2}} N m \epsilon^{2} \omega_{p}^{2}|\gamma|^{2} .
$$

Clearly, this energy must be supplied by the kinetic energy of the equilibrium distribution, so a model that predicts an unmodified equilibrium cannot be consistent.

The resolution of this paradox lies in the inadequacy of the eigenvalue dominance approximation. To see this, we include the Case-Van Kampen continuum in $f^{(0)}$ with

$$
\begin{aligned}
f^{(0)}= & \gamma g_{n} F^{\prime} e^{i n x-i n v_{0} t} \\
& +\int_{-\infty}^{+\infty} A(v) \phi_{v}(\mu) e^{i n x-i n v t} d v+\text { c.c. }
\end{aligned}
$$

where $\phi_{\nu}$ is the usual continuum singular eigenmode, ${ }^{3}$ and $A(v)$ is the amplitude of the continuum. As $t \rightarrow \infty$, the continuum term in $f^{(0)}$ vanishes (Landau damping). ${ }^{1-3}$ However, suppose we insert (24) into the right-hand side of Eq. (12) before taking the limit $t \rightarrow \infty$. We obtain, only considering the terms independent of $x$ and using $L\left(g_{n} F^{\prime}\right)=L\left(\phi_{v}\right)=1$,

$$
\begin{aligned}
L\left(f^{(0)}\right) \frac{\partial f^{(0)}}{\partial v}= & \frac{i \omega_{p}^{2}}{n}\left[\left(\gamma e^{-i n v_{0} t}\right.\right. \\
& \left.\times \int_{-\infty}^{+\infty} A^{*} \frac{\partial \phi_{v}}{\partial v} e^{i n v t} d v-\text { c.c. }\right) \\
& +\left(\gamma^{*} \frac{\partial \phi_{0}}{\partial v} e^{i n v_{0} t} \int_{-\infty}^{+\infty} A e^{-i n v t} d v-\text { c.c. }\right) \\
& +\left(\int_{-\infty}^{+\infty} \int_{-\infty}^{+\infty} A(v) A^{*}\left(v^{\prime}\right) \frac{\partial \phi_{v}}{\partial v}\right. \\
& \left.\left.\times e^{i n\left(v^{\prime}-v\right) t} d v d v^{\prime}-\text { c.c. }\right)\right] \equiv D(v, t),
\end{aligned}
$$

where

$$
\phi_{0}=g_{n} F^{\prime} .
$$

We note the crucial fact that this term, while space independent, does depend on $t$. The contribution of $D(v, t)$ to $f^{(1)}$, call it $f_{D}^{(1)}$, is given by the equation 


$$
\frac{\partial f_{D}^{(1)}}{\partial t}=-D(v, t)
$$

or

$$
f_{D}^{(1)}=-\int_{0}^{t} D\left(v, t^{\prime}\right) d t^{\prime}
$$

so that asymptotically, the modification of the equilibrium distribution is given by

$$
\lim _{t \rightarrow \infty} f_{D}^{(1)}(v, t) \text {. }
$$

This limit exists and, we note, contributes a perturbation to the energy proportional to $\epsilon^{2}$ (since $f^{(1)} \sim \epsilon^{2}$ ). A complete analysis of continuum-eigenvalue interference is presently being carried out, and will be the subject of a future paper. However, to indicate how the analysis proceeds, consider the contribution to $f_{D}^{(1)}$ of only the first two terms of $D(v, t)$. [Call this contribution $D_{1}(v, t)$. The third term is analyzed in a completely analogous fashion.]

If we write

$$
\begin{aligned}
& A(v)=B(v)+i C(v), \\
& \gamma=\theta+i \mu,
\end{aligned}
$$

and evaluate Eq. (26a), we find the contributions to $f_{D}^{(1)}$ arise from the following terms (call them $f_{1}^{(1)}$ ):

$$
\begin{aligned}
f_{1}^{(1)}(v, t)= & -2 \omega_{p}^{2} \int_{-\infty}^{+\infty} d v \frac{\partial \phi_{v}}{\partial v}([\mu B(v)-\theta C(v)] \\
& \times \frac{-\sin n\left(v-v_{0}\right) t}{n\left(v-v_{0}\right)}+[\theta B(v)+\mu C(v)] \\
& \left.\times \frac{\cos n\left(v-v_{0}\right) t-1}{n\left(v-v_{0}\right)}\right) \\
& +2 \omega_{p}^{2} \frac{\partial \phi_{0}}{\partial v} \int_{-\infty}^{+\infty} d v([\mu B(v)-\theta C(v)] \\
& \times \frac{-\sin n\left(v-v_{0}\right) t}{n\left(v-v_{0}\right)} \\
& \left.+[\theta B(v)+\mu C(v)] \frac{\cos n\left(v-v_{0}\right) t-1}{n\left(v-v_{0}\right)}\right) .
\end{aligned}
$$

Now taking the limit $t \rightarrow \infty, \sin n\left(v-v_{0}\right) t /$ $n\left(v-v_{0}\right) \rightarrow \pi \delta\left(v-v_{0}\right)$, and the terms containing this factor cancel. Similarly, ${ }^{20}$

$$
\lim _{t \rightarrow \infty} \int f(x) \frac{\cos x t-1}{x} d x=P \int \frac{f(x)}{x} d x,
$$

so the remaining terms give the result

$$
\begin{aligned}
\lim _{t \rightarrow \infty} f_{1}^{(1)}= & -\frac{2 \omega_{p}^{2}}{n} P \int[\theta B(v)+\mu C(v)] \\
& \times\left(\frac{\partial \phi_{0}}{\partial v}-\frac{\partial \phi_{v}}{\partial v}\right) \frac{d v}{v-v_{0}}
\end{aligned}
$$

A similar expression is obtained for $f_{2}^{(1)}$, the contribution to $f_{D}^{(1)}$ from the third term of Eq. (25), and we obtain

$$
\begin{aligned}
\lim _{t \rightarrow \infty} f_{2}^{(1)}= & \frac{2 \omega_{p}^{2}}{n} P \iint\left[B(v) B\left(v^{\prime}\right)+C(v) C\left(v^{\prime}\right)\right] \\
& \times \frac{\partial \phi_{v}}{d v} \frac{d v d v^{\prime}}{v-v^{\prime}} .
\end{aligned}
$$

The contribution to the kinetic energy because of $f_{D}^{(1)}$ can easily be calculated:

$$
W_{T}=m N \pi \int_{-\infty}^{+\infty} v^{2} f^{(1)}(v) d v .
$$

Writing $^{3,5}$

$$
\phi_{\nu}(v)=-\eta(v) /(v-v)+\lambda(v) \delta(v-v),
$$

a straightforward calculation gives

$$
\begin{aligned}
W_{r}^{(1)}= & -4 \omega_{p}^{2} m N \pi P \int_{-\infty}^{+\infty} \int_{-\infty}^{+\infty} \frac{B(v) B\left(v^{\prime}\right)+C(v) C\left(v^{\prime}\right)}{v-v^{\prime}} \\
& \times v^{\prime} \lambda\left(v^{\prime}\right) d v d v^{\prime}-4 \omega_{p}^{2} m N \pi P \\
& \times \int_{-\infty}^{+\infty} \frac{\theta B(v)+\mu C(v)}{v-v_{0}} v \lambda(v) d v .
\end{aligned}
$$

This loss of kinetic energy from the equilibrium distribution supplies the electric field energy, Eq. (23), of the nonlinear oscillation.

\section{DISCUSSION}

Starting from the knowledge that the solution of the nonlinear Vlasov equation enjoys global stability, we have been able to obtain analytical expressions for the saturated amplitude and phase shift of the nonlinear oscillations. Although the mathematical treatment was based on some assumptions whose physical justification is not immediately obvious (e.g., regularizing integrals "off axis" and the model of eigenvalue dominance), we have at least been able to give arguments indicating that these assumptions are plausible. For the regularization, we were able to show that the amplitude and phase shift were independent of the specific model chosen, while for the eigenvalue dominance, we were able to show that interactions with the continuum could lead to energy conservation without affecting the amplitude and phase shift (by modifying the equilibrium distribution).

A somewhat surprising result obtained is that a linearly stable collisionless plasma does not oscillate, i.e., nonlinear effects damp the oscillations. Thus, oscillation occurs only for linearly unstable regimes.

An open question is the behavior of a plasma with a degenerate root on the real axis. ${ }^{5}$ Such a plasma is predicted by a linear theory to have a secular term, proportional to some power of $t$ (Ref. 5). Nonlinear effects presumably stabilize this behavior, but so far we have not been able to see how.

\section{APPENDIX: CANONICAL REGULARIZATION}

The canonical regularization used in this paper is just a straightforward generalization of the principal value integral often encountered in physics.

If we regularize the integral $\int_{-\infty}^{+\infty}[f(x) / x] d x$ [where $f(x)$ is smooth] by going "off axis," i.e., replacing $x$ in the denominator by $x \pm i \eta$ and taking the limit $\eta \rightarrow 0^{+}$, we obtain

$$
\lim _{\eta \rightarrow 0^{+}} \int \frac{f(x)}{x \pm i \eta} d x=P \int \frac{f(x)}{x} d x \mp \pi i f(0) .
$$

The principal value integral can, of course, be evaluated by

$$
P \int \frac{f(x)}{x} d x=\lim _{\epsilon \rightarrow 0^{+}}\left(\int_{-\infty}^{-\epsilon} \frac{f(x)}{x} d x+\int_{\epsilon}^{\infty} \frac{f(x)}{x} d x\right) .
$$


Substituting $x=-y$ in the first term yields

$$
\begin{aligned}
P \int \frac{f(x)}{x} d x & =\lim _{\epsilon \rightarrow 0^{+}}\left(\int_{\infty}^{\epsilon} \frac{f(-y)}{y} d y+\int_{\epsilon}^{\infty} \frac{f(x)}{x} d x\right) \\
& =\int_{0}^{\infty} \frac{f(x)-f(-x)}{x} d x .
\end{aligned}
$$

The canonical regularization given in Eqs. (20) generalize this expression to cases where the integrand has a pole of order larger than one. These expressions are a natural consequence of the limiting procedure.

\section{ACKNOWLEDGMENTS}

One of the authors (P.F.Z.) wishes to thank Professor A. Simon and Dr. E. Larsen for useful discussions.

This work was supported by the U. S. Department of Energy under Grant No. DE-AS05-80ER10711.

${ }^{1}$ L. Landau, J. Phys. Moscow 10, 25 (1946).

${ }^{2}$ N. G. van Kampen, Physica (Utrecht) 21, 949 (1955).
${ }^{3}$ K. M. Case, Ann. Phys. (NY) 7, 349 (1959).

${ }^{4}$ F. C. Shure, Ph.D. dissertation, University of Michigan, 1963.

${ }^{5}$ M. D. Arthur, W. Greenberg, and P. F. Zweifel, Phys. Fluids 20, 1296 (1977); see also M. D. Arthur, Ph.D. dissertation, Virginia Polytechnic Institute and State University, 1979.

${ }^{6} \mathrm{~J}$. Cooper and A. Klimas, J. Math. Anal. Appl. 75, 306 (1980)

${ }^{7}$ W. E. Drummond and D. Pines, Ann. Phys. 28, 478 (1964).

${ }^{8}$ E. Frieman, S. Bodner, and P. Rutherford, Phys. Fluids 6, 1298 (1963).

${ }^{9}$ E. Frieman and P. Rutherford, Ann. Phys. 28, 134 (1964).

${ }^{10} \mathrm{~A}$. Simon and M. N. Rosenbluth, Phys. Fluids 19, 1567 (1976).

"'T. O'Neil, Phys. Fluids 8, 2255 (1965).

${ }^{12}$ S. P. Auerbach, Phys. Fluids 20, $1836(1977)$

${ }^{13}$ S. Paveri-Fontana, B. L. Willis, and P. F. Zweifel, Trans. Theory Stat. Phys. 10, 137 (1981).

${ }^{14}$ P. F. Zweifel and J. Ohlmann, Prog. Nucl. Energy 8, 145-150 (1981).

${ }^{15}$ G. Spiga, B. L. Willis, and P. F. Zweifel, Trans. Theory Stat. Phys. 10, 149 (1981).

${ }^{16} \mathrm{G}$. Spiga and B. D. Ganapol (private communication).

${ }^{17}$ C. M. Bender and S. A. Orszag, Advanced Mathematical Methods for Scientists and Engineers (McGraw Hill, New York, 1978), Chap. 7.

${ }^{18}$ I. M. Gel'fand and G. E. Shilov, Generalized Functions (Academic, New York, 1964), Vol. I, p. 61.

${ }^{19}$ E. W. Larsen, C. Burnap, and P. F. Zweifel, Transp. Theory Stat. Phys. 12, 73 (1983).

${ }^{20}$ T. Apostol, Mathematical Analysis, 2nd ed. (Addison-Wesley, Reading, MA, 1974), p. 314. 